\title{
Intelligent Mobility Management Model for Heterogeneous Wireless Networks
}

\author{
Sanjeev Prakash \\ ${ }^{1}$ Department of Computer Science \\ and Engineering, Sant Longowal \\ Institute of Engineering and \\ Technology, Longowal, Punjab, \\ India
}

\author{
R B Patel \\ Dept. of Computer Science and \\ Engineering \\ Chandigarh College of Engineering \\ and Technology \\ Chandigarh, India
}

\author{
V. K. Jain \\ Department of Electrical and \\ Instrumentation Engineering, Sant \\ Longowal Institute of Engineering \\ and Technology, Longowal, Punjab, \\ India
}

\begin{abstract}
Growing consumer demands for access of communication services in a ubiquitous environment is a driving force behind the development of new technologies. The rapid development in communication technology permits the end users to access heterogeneous wireless networks to utilize the swerve range of data rate service "anywhere any time". These forces to technology developers to integrate different wireless access technologies which is known as fourth generation (4G). It is become possible to reduce the size of mobile nodes (MNs) with manifold network interfaces and development in IP-based applications. 4G mobile/wireless computing and communication heterogeneous environment consist of various access technologies that differ in bandwidth, network conditions, service type, latency and cost. A major challenge of the $4 \mathrm{G}$ wireless network is seamless vertical handoff across the heterogeneous wireless access network as the users are roaming in the heterogeneous wireless network environment. Today communication devices are portable and equipped with manifold interfaces and are capable to roam seamlessly among the various access technology networks for maintaining the network connectivity, since no single-interface technology provides ubiquitous coverage and quality-of-service (QoS).

This paper reports a mobile agent based heterogeneous wireless network management system. In this system agent's decision focuses on multi parameter system (MPS). This system works on the parameters- network delay, received signal strength, network latency and study of the collected information about adjoining network cells viz., accessible channel. System is simulated and a comparative study is also made. From results it is observed that system improves the performance of wireless network.
\end{abstract}

\section{Keywords-FNS; MNS; MN; WLAN; Mobile Agent}

\section{INTRODUCTION}

The adoption of wireless technology is increasing rapidly from few decades and has become popular means for computing and communication to access the information even the users are away from their home network. The driving force behind this development is basically portability of hand held devices viz., Smart mobile phones, tablets, Laptops and Personal Digital Assistances (PDA), etc. These devices are equipped with multiple interfaces due to availability of several wireless technologies and mobile applications. To meet the increasing demand of computing and communication services in ubiquitous environment there is need to integrate different emerging wireless access technologies together which is known as Fourth Generation (4G) wireless computing and communication system [1, 12]. This wireless system is popular due to its heterogeneity. A heterogeneous wireless network generally is an integration of fixed backbone network and wireless networks. Mainly three distinct sets of entities play key role in the system, viz., mobile nodes (MNs), Mobile Network Stations (MNSs) and fixed network stations (FNSs). A moving network station (NS) which retains its network connection is called a MNS. A fixed network consists of FNSs and communication links among FNSs and none moving nodes or devices. Some of the FNSs are designated as base stations (BSs). A BS is augmented with wireless interfaces and works as a gateway for communication among the fixed and wireless networks. Communication range of wireless transreceivers limits the MNS/MN to communicate with a BS. The average covering area of a region is of the order of $1 \mathrm{KM}$ in radius [2].

A handoff management is required when a user moves from one wireless cell to another, abandoning the connection with one point of attachment to another [15]. When a handoff occurs within the domain of a homogeneous wireless access technology occurring event is known as horizontal handoff and when this event occurs among heterogeneous wireless access network technologies is known vertical handoff. Horizontal handoff occurs when the MNs are moving far from point of attachment and enter into the low signal strength area in a homogeneous wireless network. In a heterogeneous environment, users have an opportunity to access the different technologies networks. A user may be benefited from different network characteristics (coverage, bandwidth, latency, power consumption, cost, etc.) which are not comparable directly [18]. MN mobility is supported by vertical handoff as the communication technology and access supporting infrastructure change. Sometime vertical handoff takes place due to user's convenience rather than unavailability of connection [17][24]. The handoff process becomes more complex in such an environment compared to the homogeneous one.

Vertical handoff event comprises three handoff steps: 1) initiation 2) decision 3) execution. Network and user related information are collected during the handoff initiation, this step is also known as system discovery, system detection, handover information gathering, and handoff initiation. Handoff decision stage plays an important role and is one of 
critical process of handoff. It is also known as network selection or system selection. Handoff execution is either hard handoff or soft handoff.

The constantly changing environment is demanding for more and more services. In such a condition to maintain the required grade of services in a region is a driving force for the technology developer to split a region for handling the traffic increased without increasing the bandwidth of the system [3]. A BS is accountable for forwarding information and voice packets among a MNS/MN and a fixed network. To achieve the required goal a MNS/MN may cross the boundary among two regions while it is in conversation. The job of forwarding information/voice packets among the fixed network and the MNS/MN may be routed through the new regions for facilitating the end-to-end links in the dynamically network topological changing environment. Heterogeneous wireless network facilitates users to access the diverse range of access network technologies which are differing in coverage range, bandwidth, throughput, latency and cost [14]. To provide a seamless connectivity among these heterogeneous technologies an efficient mobility management framework is needed that facilitates roaming to users from one network to another [4][5][6][7].

Thus, researchers are continuously making their efforts for developing a common platform for the heterogeneous wireless computing and communication networks to offer secure, seamless, and required bandwidth connectivity to users [8][9][11][16]. The rest of this paper is organized as follows. In the section II system architecture is described. Registration management protocol and handoff management protocol are presented in the section III and IV respectively. simulation and performance study is presented in section V. Finally, conclusion and future work is described in the section VI.

\section{SYSTEM ARCHITECTURE}

It is assumed that global wireless mobile communication network is divided into network domains (mobile switching centre-MSC), regions (sub-networks, i.e., base switching centre (BSC) controlled network) and Mobile Network Stations (MNSs) as shown in Figure 1. A MSC works as a network management server (NMS) in each network domain and keeps information other existing NMSs in the global wireless mobile communication system. A NMS behaves like a Home Subscriber Server (HSS) which houses subscribers profiles. It is also known as subscriber profile repository and formally known as home location register (HLR). It maintains the current position of all the MNs which are registered in that network domain or transited through. This component work as a carried forward for UMTS and GSM and is a central database which is consisting of information regarding all the available MSCs (network operator's subscribers). Other component of a NMS is Packet Data Network Gateway (PDNG) which communicates with the outside world, it works similar to packet data networks (PDNs), using SGi interface [10]. Each data packet network is identified by BS and MAC address of the MN. The PDNG act as a GPRS support node (GGSN) and providing GPRS support node (SGSN) for UMTS and GSM. A BSC is also known as serving gateway (SGW) and play similar role as a router, and forwards information packets among a BS and a PDNG. The function of mobility management entity (MME) is to control the sophisticated process of the mobility using signalling messages and HSS. NMS also implements the Policy Control and Charging Rules (PCRs) and is accountable for policy control and management. It implements flow-based charging functionalities.

NMS is also equipped with Policy Traffic Switch (PTS) for identifying the location at which it intersects the traffic. It embeds a subscriber policy broker (SPB) and a service delivery engine (SDE) in the data plane of any network. It may be physical or virtual, with any combination of access technologies. Embedded within the PTS, the SDE makes policy decisions locally and prevents unnecessary signalling, reduces the load on NMS, and delivers faster decisions. SPB works like as single data warehouse for subscriber's information. SDE makes system to operate in heterogeneous environment.

A NMS also maintains information about all the available BSCs. A BSC defines a boundary of a region. It maintains unique name of each BSCs (regions). It identifies the region/BSC in/under which a MNS/MN is currently available.

A region (BSC) maintains information about all the available $\mathrm{BSs} / \mathrm{MNSs}$ in a region. A MN may be a member of an available MSC/BSC covered area/region or may register in a new region (MSC/BSC). In a region, an authentication authorization access server (AAAS) is used to maintain database of users presently available at a BS. It works like a gateway (BSC) of a subnetwork. It contains information of location of each MNS/MN which are registered in that region or transited through it. It works like a visitor location register (VLR). This network station (NS) also acts as the Mobile Node Name Server (MN2S). It maintains unique name of all MNSs/MNs, registered in a particular region. When a new $\mathrm{MNS} / \mathrm{MN}$ is registered it details are registered in the AAAS of its birth region.

\section{Registration MANAGEMENT PROTOCOL}

HSS uses a tuple of three attributes in the format $(M N, F D, r)$ to stores the information in its database. In this tuple FD stands for foreign network domain and $r$ stands for a region. It signifies that a $M N$ may be present in the $r$ of the $F D$ or transited through it. A tuple of three attributes $(M N, r, N i l)$ entered in AAAS shows that $M N$ is available in region $r$ or transited through it. A tuple in form $(M N, N i l, M N S)$ signifies that a $M N$ exists in that region and is at a particular BS/MNS. The name/id of $M N$ is used as a primary key for NMS and MN2S.

A MN movement among network domains is always achieved through the NMS. An inter domain movement of a MN updates location in HSS of the current network domain and registers in the HSS of the destination network domain.

In intra region movement, a $\mathrm{MN}$ updates its current position in the AAAS of the region which is called as an Intra Region Location Update. When inter region movement takes 
place. A MN updates the position information in the AAAS of current region and registers in AAAS of the destination region. It specifies a BS/MNS in that region to which it is travelling. This protocol uses three processes to deal with MNs: Identification (Id) attachment, movement and location update in the lifetime of a MN. In total this operation is called a particular phase for a MN movement. The protocol defines four atomic operations on home location register SPR (HSPR) and visitor location register SPR (VSPR). (a) Id attachment process is executed for naming a newly registered $\mathrm{MN}$, whose birth location is also stored. This process signifies the insertion of a new tuple in the database. This process fails if a tuple with the same Id already exists in the database. (b) newloc process is executed when a MN changes its position, by moving to a new location. It updates the tuple already available in the database. (c) Find process is executed when interaction with a $\mathrm{MN}$ is required. For a given $\mathrm{MN}$ name, it returns the current location of the $\mathrm{MN}$. (d) Id detachment process is executed when a $\mathrm{MN}$ id is no longer used (i.e., the MN has been disposed off). This process deletes the related tuple from the database.

Each NMS, BSC, BS and MNS are equipped an intelligent agent and a mobile agent (MA)[25] for maintaining network topology and current status of its neighbouring BSs. Normally agents observe technique for order preference by similarity to the ideal solution (TOPSIS) for getting the

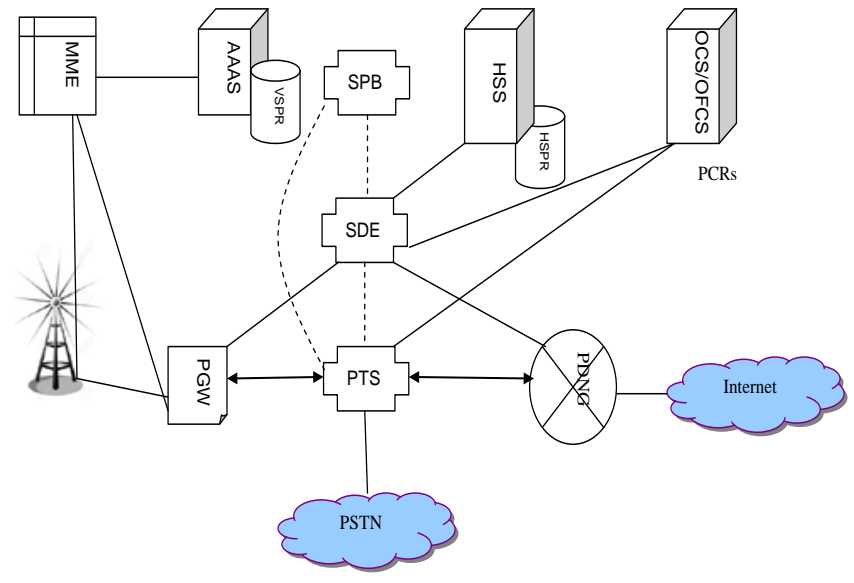

Fig. 1. Heterogeneous Wireless Network Environment Distribution Policy

services and network path from one point to another. In TOPSIS the best alternative is a shortest distance from the positive ideal key and the farthest distance from the negative ideal key. A matrix is constructed on the basis of set of alternative and corresponding attributes (criteria). In this method it is assumed that there are $A_{m}$ alternatives and $C_{m}$ criteria [23].

\section{HANDOFF MANAGEMENT PROTOCOL}

In a heterogeneous wireless networks there are diverse access technology and each have different transmission power, bandwidth, security and cost. Thus, a single attribute is not sufficient to take handoff decision to provide a desired QoS. In heterogeneous wireless network environment, a dynamic negotiation helps to improve its performance of the network for dynamic negotiation mobile agents technology play the key role. The goal of negotiation is to maximize the utility of a future decision. Each cell acquires a free channel for establishing a connection. In a given limit it requires to achieve a target. Negotiation stops as soon as defined limit is attained. A cell goes through this stage, when all channels are occupied except reserved channels. During this stage a mobile agent uses message exchange protocol for gathering the information about the channels status. In this stage it concludes for avoiding the handoff call blocking probability. In decision agent focuses on multi parameter systems (MPS). This system works on the parameters- network delay, received signal strength, and study of the collected information about adjoining network cells viz., accessible channel. Mobile agent sitting on the MSC/BSC/BS/MNS executes the following algorithm to update the candidate's network in database.

$$
\begin{gathered}
\text { if }\left(\mathrm{RSS}_{\text {current }}-\mathrm{RSS}_{\mathrm{Th}}\right)<0 \\
\text { search for another network } \\
\text { endif } \\
\text { if }\left(\mathrm{RSS}_{\text {new }}-\mathrm{RSS}_{\mathrm{Th}}\right)>0 \\
\text { update the network database } \\
\text { endif }
\end{gathered}
$$

A mobile execute the algorithm shown in Figure 2. After certain interval a $\mathrm{MN}$ reads the available network database in the area. If a network is there in the area then $\mathrm{MN}$ stay connected with available network and it checks regularly the network database within coverage area. If a network is not available in the coverage area then it terminates the process. If there is more than one available network then TOPSIS is used to arrange the network in an order. If the application priority is higher and bandwidth requirement is higher and low cost network is required then a $\mathrm{MN}$ chooses handoff to the alternative-1. If application priority is low and bandwidth requirement is higher and low cost network is required the $\mathrm{MN}$ goes handoff to the alternative-2. And if low bandwidth or low cost is required then the $\mathrm{MN}$ selects handoff to the alternative-3.

\section{Simulation AND PERFORMANCE StUdy}

For the deployment of agents in the presented model, a MATLAB and its Java features, MAC-SF [23] and PMADE agent framework [25][26] are used to study the agent migration under different network load conditions. To study the agent migration, three networks each having twenty one nodes are used. These networks are connected through one gateway. Main control frames are modified for simulating the essential measurement techniques and included the suitable information. The parameters communication cost, network bandwidth requirement of each $\mathrm{MN}$, etc. are taken into consideration. This alteration does not modify the functioning of the system. A heterogeneous wireless network environment setup is created using the following technologies- WLAN, WiMAX [13], and GSM. Figure 3 shows the simulation scenario of the network used to simulate the agent guided distance based scheme and RSS scheme. The simulation network consists of minimum of 4 BS (GSM), 3 MNSs, 21 WLAN APs and 1 WiMAX point against $1 \mathrm{BS} / 1 \mathrm{MNS}$ and 3 APs. A minimum number of 200 MNs are considered. This 
number may increase or decrease because of the dynamic nature of the MNs. BS's/MNSs signals will be in overlapping fashion. In simulation cell radius is fixed to $1 \mathrm{KM}$. It is assumed that cells have equal bandwidth capacity and it is 10 $\mathrm{MHz}$.

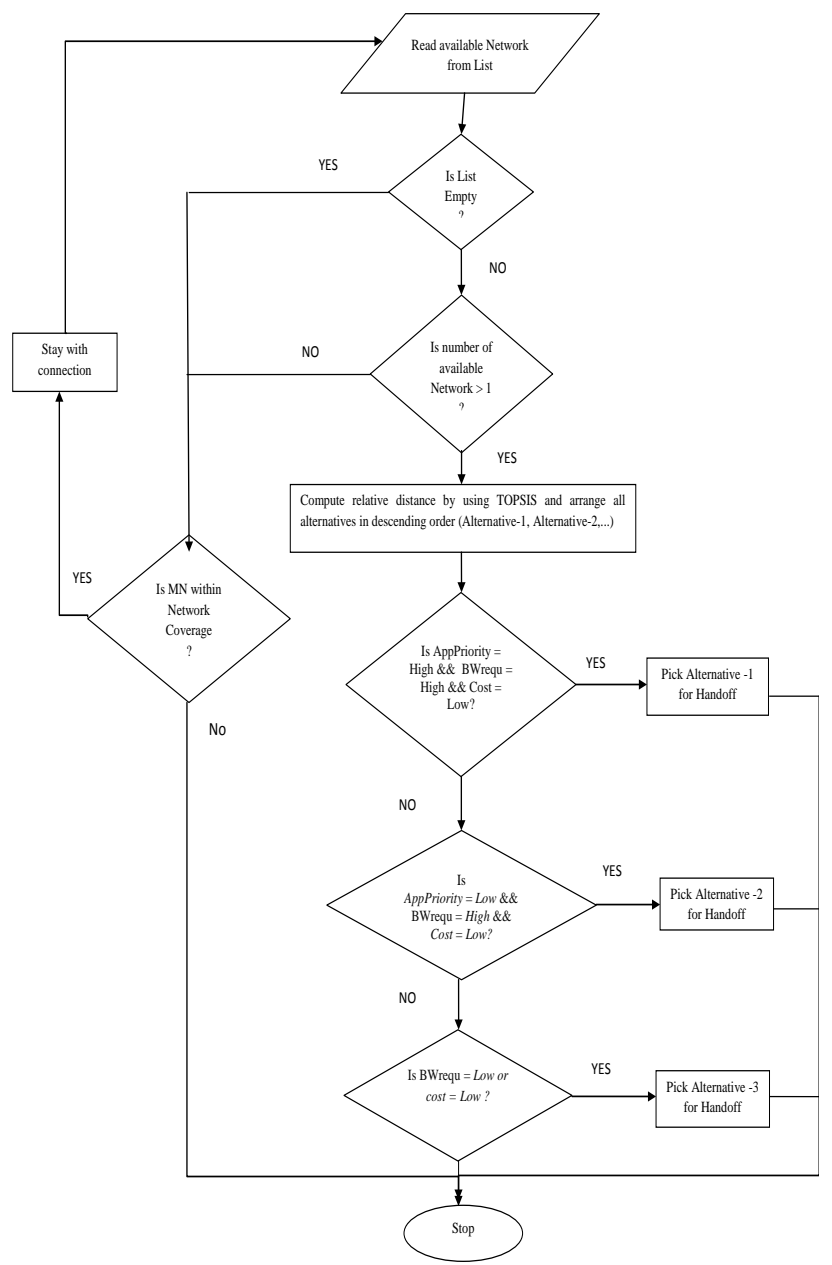

Fig. 2. A Multi Parameter Algorithm for Handoff

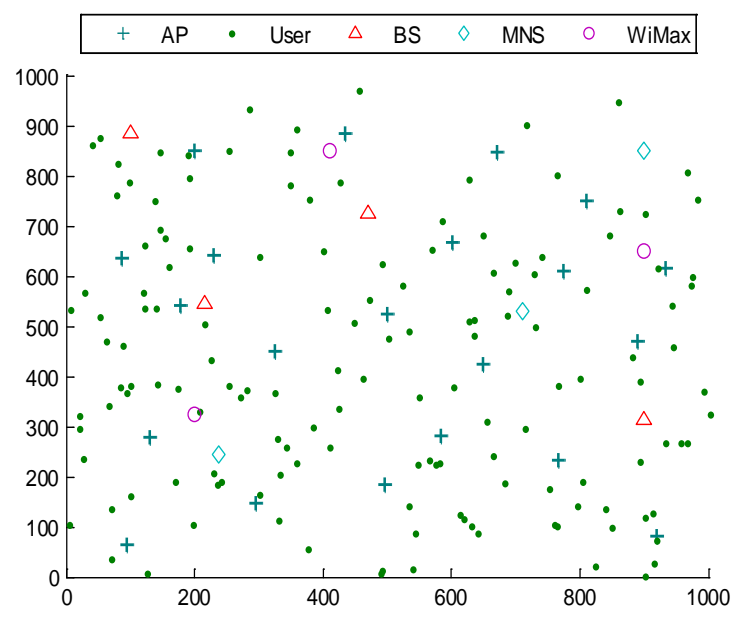

To avoid the boundary effect of a network size a wraparound edge cells are used. This ensures that the number of adjoining cells for a cell varies between three and six. This guarantees that there is always a network available for the communication. In the system there may be some areas which will be accessible by two or more networks. This covering helps the user to understand the network choice procedure in the presence of customer preferences.

The parameters given in Table 1 are used in the simulation. It is assumed that only an authenticated $\mathrm{MN}$ in the foreign networks (FNs) has a free access. it is also not taken into account authorization authentication and access $\left(\mathrm{A}^{3}\right)$ and admission control mechanisms. A $\mathrm{MN}$ is moving with a fixed speed of $1.5 \mathrm{~m} / \mathrm{s}$. In simulation it is assumed that $\mathrm{MN}$ is executing real-time as well as and non-real time applications, viz., audio/video as well as textual data. A priority is set as per the importance of applications to allow system to create single/multiple sessions.

Throughput of a network is directly related to the number of customers utilizing the link. Data transfer rates may differ upon accessible access network category, configuration, and load. A successful handoff decreases the number of conflicts on heavily loaded network. A conflict always regenerates the network traffic. Because packets are retransmitted and if this retransmission of packets reaches to the threshold value then packets are be discarded. Figure 4 presents throughput in handoff mechanism for four systems. One is the conventional handoff system [19], second is cost based system in 3rd system [20] author is using intelligent agent and 4th is using intelligent stationary and mobile agents. In the past research algorithm when a MN is moving and a new network is detected who's RSS is higher and MN switched to higher RSS network. In MPS algorithm unnecessary handoffs are avoided and selection of network is not only based on the RSS but also on considered the application types, cost and peak-hour time. Agents (stationary intelligent and mobile agents) play the key role in successful handoff process when MNs are interested in priority based services. It decreases the number of conflicts at peak hour use of system and in heavy traffic load. The probability of loss of packets increases when the conflicts occur. To avoid such situation agent technology is used and better result is observed as shown in Figure 4.

A MN requirement always depends on its capability to process the received information. Power of a $\mathrm{MN}$ is measured in terms of communication \& computing speed, memory, and power consumption against the throughput. Based on these parameters a node is being in the position to compute the requirement of the resources for the completion of the task which it wants to initiation in a network after the handoff. Further the same MN may change its expectation depending on the present context, or eventually as the network bandwidth improves and become available and/or access cost decreases. A network always announces about its usage and the available bandwidth. This information is intelligently maintained in the form of intelligent clustered database. System throughput is observed at a MN after the handoff completion.

Fig. 3. Simulation Scenario 
TABLE I. ASSUMPTION AND VALUES OF PARAMETERS

\begin{tabular}{|l|l|}
\hline Parameter & Values \\
\hline Agent Platform & PMADE 1.1 and MAC-SF \\
\hline Simulator & MLAB 15a \\
\hline Simulation Area & $1000 x 1000 \mathrm{~m}^{2}$ \\
\hline Simulation time & 200 seconds \\
\hline Mobile Nodes & 200 \\
\hline Access Points & 21 \\
\hline BS & 4 \\
\hline MNS & 3 \\
\hline Number of WiMax & 3 \\
\hline Users & 128 \\
\hline Threshold(WLAN to cellular network) & $-85 \mathrm{dBm}$ \\
\hline WLAN range & $200 \mathrm{~m}$ \\
\hline Access Point Transmitter Power (min) & $30 \mathrm{dBm}$ \\
\hline Access Point Transmitter Power (max) & $100 \mathrm{dBm}$ \\
\hline BS Transmitter Power & $33 \mathrm{dBm}$ \\
\hline Cable loss & $1.7 \mathrm{~dB}$ \\
\hline Threshold (cellular network to WLAN) & $-80 \mathrm{dBm}$ \\
\hline Channel gain power & $33 \mathrm{dBm}$ \\
\hline Antenna height of BS & $30 \mathrm{~m}$ \\
\hline BS Operating Frequency & $894 \mathrm{MHz}$ \\
\hline Bandwidth for GSM & $10 \mathrm{MHz}$ \\
\hline Maximum output power of GSM & $39 \mathrm{dBm}$ \\
\hline Data rate & $2 \mathrm{Mbps}$ \\
\hline Communication Channels & 16 \\
\hline Traffic type & Audio/Video/Text \\
\hline Transmit power & $5 \mathrm{~mW}$ \\
\hline AP capacity & $40 \mathrm{sessions}$ \\
\hline & \\
\hline
\end{tabular}

Figure 4 gives a comparative study of different approaches. After the handoff execution classic and cost based approaches are showing the average throughput for transferring $1 \mathrm{MB}$ of data. This handoff takes place between GSM and WiMAX networks. The result also shows that the presented schemes alter the networks between 1 and 6 seconds. Further, it is also observed that handoff period varies between 1 to 3 seconds. The professed throughput of MPS approach is steady till the completion of the task. The intelligent clustered database is key factor behind a handoff toward the best available network. MPS is an intelligent choice method gives the priority to network bandwidth, communication cost and continued with its activity till the end of the initiated task. Mobile agents working in the network are periodically updating the database for the selected network parameters. Thus, every network is always being with the updated and above the threshold value of the RSS. Further this property of the system removes RSS issue of connectivity. MPS improves the system throughput in comparison to cost based, classic handoff and approach given in [20]. A MN moves arbitrarily and channel fading is a function of distance. It may vary with the changes in distance among the MN and BS. It also showed that the RSS of two BS change up and down.

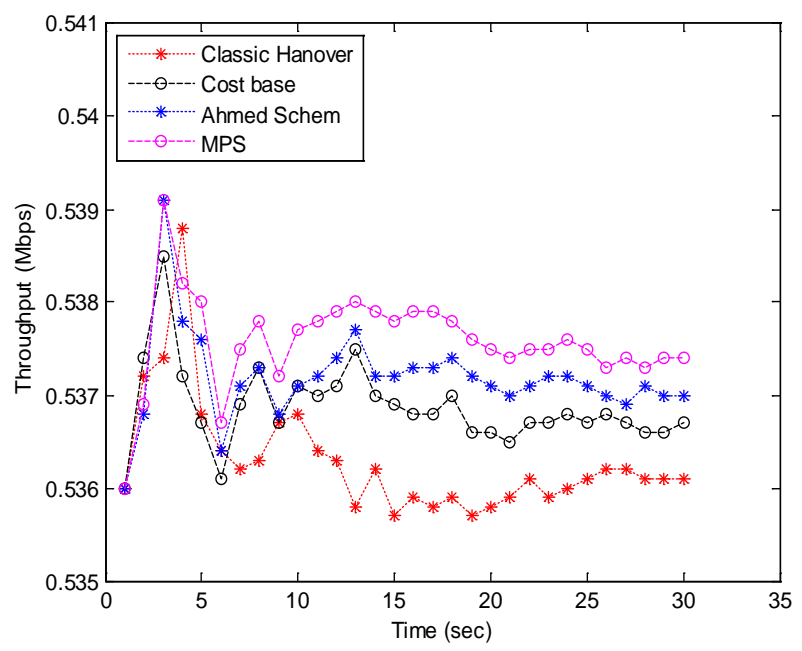

Fig. 4. Average Throughput

Figure 5 shows that the average handoffs delay for voice packet transmission while performing handoff. Handoff delay depends on the routing delay. And same is considered in the simulation while switching from one network to another. The make before-break scheme helps MPS to achieve low handoff delay. MPS improves network performance approximately by $6 \%, 13 \%$ and $21 \%$ in comparison to approach given in [20], cost-based and classic handoff schemes, respectively.

Another parameter which is important in handoff process is to compute the handoff blocking rate. It is percentage of calls which are not able finish their services. It may be due selection of an un-appropriate wireless access network or due to unavailability of the list of available wireless access networks. When a BS fails to assign a free channel an incoming call process is automatically blocked. The overall system service stability always depends on blocking rate. If a system is reflecting low blocking rate means system is efficient. TOPSIS method is used for selection of the target network which reduces the probability of blocking of mobility management protocols. This model takes into consideration updated database maintained by the mobile agents. The network topology parameter plays major role in the analysis because it gives the mobility patterns of a MN. 


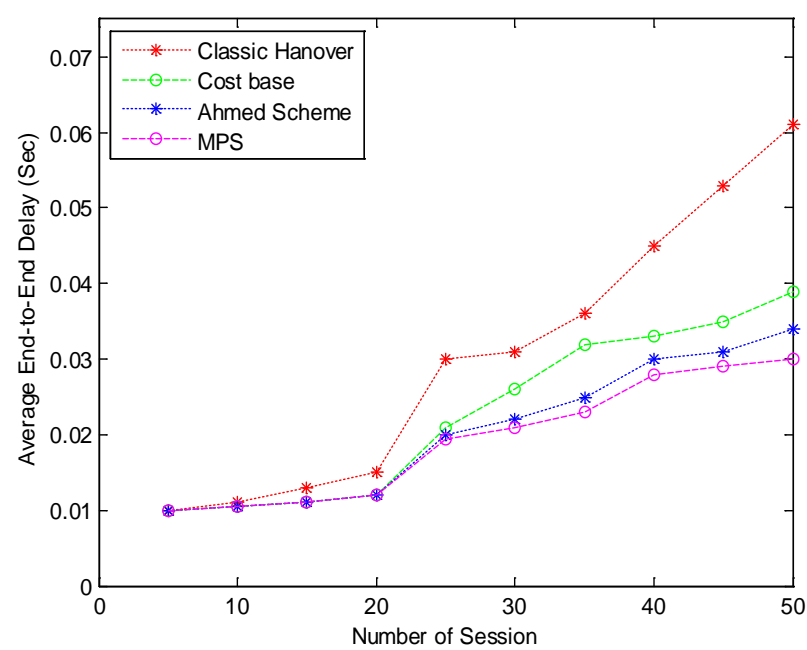

Fig. 5. Average end-to-end transmission delay

Figure 6 shows that MPS decreases handoff blocking rate in comparison to [20], classic scheme [22] and cost-based scheme [21]. In simulation it is assumed that $64 \mathrm{MNs}$ are working simultaneously. It is seen that when the number of MN are less than 25-27, all the systems behave similarly. But when this active number of MNs increases, MPS approach outperforms in comparison to classic and cost- based systems but it performs much better with [20]. There may be reason that the handoff resources, in these two (classic and costbased) systems are not adequate to satisfy all the handoff processes and better list of resources available in case of [20] to satisfy all the handoff requests. MPS steadies even after the access of more MNs.

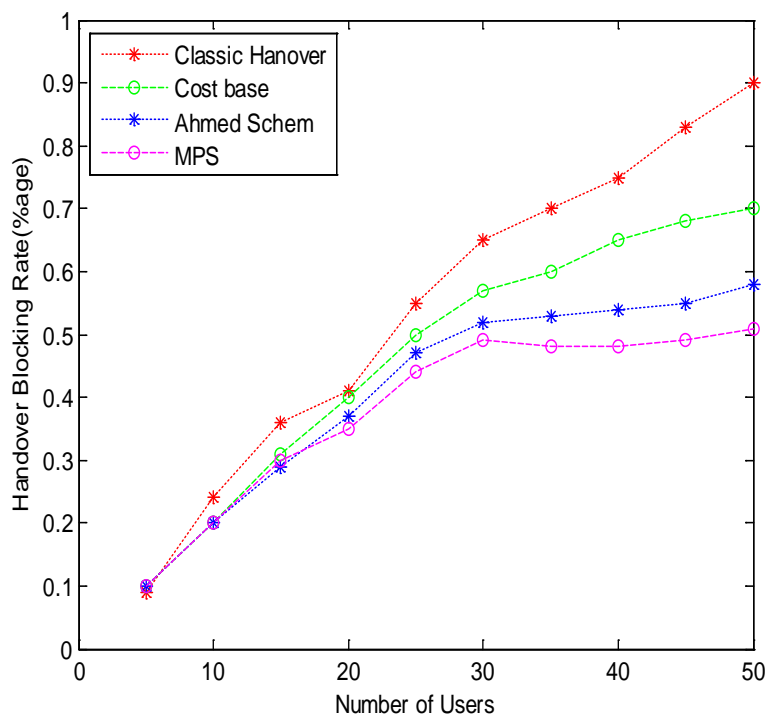

Fig. 6. Handoff blocking rate comparison

\section{CONCLUSION AND FUTURE WORK}

In this paper a mobile agent based heterogeneous wireless network environment distribution policy is presented for $4 \mathrm{G}$ networks. This is MPS based system in which handoff initiations and decisions are delegated by intelligent mobile agents. A clone of agent is required to serve a MN. A handoff is initiated by a client agent which is executed either in a $\mathrm{MN}$ or at the network side, depending on the basis of handoff generating actions. Normally it is observed that MN devices having limited battery power, computing power so, it is useful if a handoff decision takes place at the network side. MPS permits both type of access facility and core carrier services for handoff decision for better adjustment to client and demand requirements. Proposed system maintains an intelligent clustered of database of carrier service and agent for carrier selection. The system is evaluated through a simulation is carried out focusing on its impact on handoff. The results are satisfactory for the feasibility of the presented system. Future work will be focused on mobility management for high speed users.

\section{REFERENCES}

[1] Anna Marie Vegni, Gabriele Tamea, Tiziano Inzorilli and Roberto Causani, "A combined Vertical Handover Decision Metric for QOS Enhancement in next Generation Network," IEEE international conference on wireless and mobile computing, Networking and Communication, 2009.

[2] Q. Song and A. Jamalipour, "Network selection in an integrated wireless LAN and UMTS environment using mathematical Modelling and computing techniques," Wireless Communications, IEEE, 12(3), 4248, 2005.

[3] A. H. Zahran, B. Liang, and A. Saleh, "Signal threshold adaptation for vertical handoff in heterogeneous wireless networks," Mob. Netw. Appl., vol. 11, no. 4, pp. 625-640, 2006.

[4] Ben-Jye Chang and Jun-Fu Chen, "Cross-Layer Based Adaptive Vertical Handoff with Predictive RSS in Heterogeneous wireless Network," IEEE Transaction on Vehicular Technology, Vol. 57, No. 6, November 2008.

[5] Q. Yu, W. Jiang, and Z. Xiao, "3G and WLAN heterogeneous network handover based on the location information," International Conference on Communications, Circuits and Systems (ICCCAS), Nov. 2013, Vol. 2, pp. 50-54.

[6] X. Li and R. Chen, "Adaptive vertical handover algorithm based on user experience for heterogeneous network," 6th International Congress on Image and Signal Processing (CISP), December 2013, Vol. 3, pp. 15401544.

[7] A. Çalhan and C. Çeken, "Case study on handoff strategies for wireless overlay networks,” Computer Standards \& Interfaces, 35(1), 170-178, 2013

[8] Sang-Jo Yoo, D. Cypher, and N. Golmie, "Timely effective handover mechanism in heterogeneous wireless networks," Wireless Personal Communications, 52(3), 449-475, 2010.

[9] R. Verma and N.P. Singh, "GRA based network selection in heterogeneous wireless networks. Wireless personal communications, 72(2), 1437-1452, 2013.

[10] F. Bari and \& V. Leung, "Automated network selection in a heterogeneous wireless network environment,” Network, IEEE, 21(1), 34-40, 2007.

[11] X. Yan, N. Mani, and Y.A. Şekercioğlu, "A travelling distance prediction based method to minimize unnecessary handovers from cellular networks to WLANs. Communications Letters, IEEE, 12(1), 1416, 2008.

[12] S. Mohanty and I. Akyildiz, "A cross-layer (layer $2+3$ ) handoff management protocol for next-generation wireless systems," IEEE Trans. Mobile Computing, 2006.

[13] Z. Becvar, P. Mach, and B. Simak, "Improvement of handover prediction in mobile WiMAX by using two thresholds," Computer Network, vol. 55, no. 16, pp. 3759-3773, 2011. 
[14] H. Wang, R. Katz, J. Giese, "Policy-enabled handoffs across heterogeneous wireless networks," In Proceedings of $2^{\text {nd }}$ IEEE Workshop on Mobile Computing Systems and Applications, (WMCSA'99), 1999, pp. 51-60.

[15] W. Chen, J. Liu, H. Huang, An adaptive scheme for vertical handoff in wireless overlay networks, in: Proceedings on the 10th International Conference on Parallel and Distributed Systems (ICPADS 2004), 2004, pp. 541-548.

[16] O. Ormond, J. Murphy, G. Muntean, “Utility-based intelligent network selection in beyond 3G systems," IEEE International Conference on Communications (ICC 2006), vol. 4, pp. 1831- 1836.

[17] B.-J. Chang and J.-F. Chen, "Cross-layer-based adaptive vertical handoff with predictive RSS in heterogeneous wireless networks,” IEEE Trans. Veh. Technol., 2008.

[18] B.-J. Chang, J.-F. Chen, C.-H. Hsieh, and Y.-H. Liang, "Markov decision process-based adaptive vertical handoff with RSS prediction in heterogeneous wireless networks,” in IEEE Wireless Communications and Networking Conference, 2009, pp. 1-6.

[19] J. Raiyn, “A Novel Handover Scheme Based on Adaptive Agent for Reducing Real-Time Communication Latency in Automation Environment,” EUROSIM, pp. 555-561, April, 2008.

[20] Atiq Ahmed, Leïla Merghem-Boulahia, and Dominique Gaïti. "An intelligent agent-based scheme for vertical handover management across heterogeneous networks," annals of telecommunications-annales des telecommunications 66, no. 9-10,pp. 583-602, 2011.
[21] W. Yifei, L. Xiaowei, S. Mei, S. Junde, "Cooperation radio resource management and adaptive vertical handover in heterogeneous wireless networks". In: International conference on natural computation, vol 5,2008, IEEE Computer Society, Los Alamitos, pp 197-201.

[22] Ylianttila M, Mäkelä J, Pahlavan K, “Analysis of handoff in a locationaware vertical multi-access network,” Computer Network, 47:185-201, 2005.

[23] Sanjeev Prakash, R. B. Patel and V. K. Jain, "Movement Assisted Component Based Scalable Framework For Distributed Wireless Networks", International Journal on Computational Science \& Applications (IJCSA) Vol.5, No.5, pp. 71-86, 2015.

[24] M. Kassar, B. Kervella, and G. Pujolle, "An overview of vertical handover decision strategies in heterogeneous wireless networks", Computer Communications, 31(10), pp.2607-2620,2008.

[25] R. B. Patel, K. Garg, "PMADE - A Platform for Mobile Agent Distribution \& Execution," in Proceedings of 5th World MultiConference on Systemics, Cybernetics and Informatics (SCI2001) and 7th International Conference on Information System Analysis and Synthesis (ISAS 2001), Orlando, Florida, USA, July 22-25, 2001, Vol. IV, pp. 287-292.

[26] R. B. Patel and K. Garg, "A New Paradigm for Mobile Agent Computing," WSEAS Transaction on Computers, 1(3): 57-64, Jan. 2004. 